\title{
EXPONENTIAL SUMS AND NEWTON POLYHEDRA
}

\author{
ALAN ADOLPHSON AND STEVEN SPERBER
}

Let $p$ be a prime number and let $k$ denote the field of $q=p^{a}$ elements. Fix a nontrivial additive character $\Psi: k \rightarrow \mathbf{Q}\left(\zeta_{p}\right)^{\times}$. Given a variety $V$ of dimension $n$ and a regular function $f$ on $V$, with both $V$ and $f$ defined over $k$, one can define an exponential sum

$$
S(V, f)=\sum_{x \in V(k)} \Psi(f(x))
$$

where $V(k)$ denotes the $k$-rational points of $V$. It is a classical problem to find conditions on $V$ and $f$ that will imply a good estimate for $|S(V, f)|$. By "good estimate" we mean an inequality of the form

$$
|S(V, f)| \leq C \sqrt{q}^{n},
$$

where $C$ is a constant depending on $V$ and $f$ but not on $q$.

Deligne's fundamental theorem [3] reduces the problem of estimating the archimedean size of exponential sums to the problem of computing certain associated $l$-adic cohomology groups. Let $\mathbf{A}^{n}$ denote affine $n$-space over $k$ and let $\left(\mathbf{G}_{m}\right)^{n}$ denote the product of $n$ copies of the multiplicative group $\mathbf{G}_{m}$ over $k$. The purpose of this note is to report on some general criteria, when $V=\left(\mathbf{G}_{m}\right)^{n}$ or $\mathbf{A}^{n}$, that allow us to calculate this cohomology and hence obtain sharp archimedean estimates for the corresponding exponential sums. These same criteria allow us to obtain apparently sharp $p$-adic estimates for the exponential sums as well, although space limitations prevent us from describing them here. Connections between the $p$-adic theory and Newton polyhedra already appear in [7 and 8].

A novel feature of our work is the use of Dwork cohomology $[4,5]$ to compute $l$-adic cohomology. The results of this note have not so far been obtainable by purely $l$-adic methods. Complete proofs and references will appear elsewhere. We are indebted to B. Dwork and N. Katz for many helpful discussions.

1. Statement of results. Let $k_{r}$ denote the extension of $k$ of degree $r$ and let $\operatorname{Tr}_{r}: k_{r} \rightarrow k$ be the trace map. Let $\bar{k}$ denote the algebraic closure of $k$. Set

$$
S_{r}(V, f)=\sum_{x \in V\left(k_{r}\right)} \Psi\left(\operatorname{Tr}_{r} f(x)\right)
$$

Received by the editors November 1, 1986.

1980 Mathematics Subject Classification (1985 Revision). Primary 11L40; Secondary 14F20, 14F30.

First author partially supported by NSF Grant No. DMS-8401723; second author partially supported by NSF Grant No. DMS-8301453. 
where $V\left(k_{r}\right)$ denotes the $k_{r}$-rational points of $V$. Define the associated $L$ function $L(V, f ; t)$ by

$$
L(V, f ; t)=\exp \left(\sum_{r=1}^{\infty} S_{r}(V, f) t^{r} / r\right) \in \mathbf{Q}\left(\zeta_{p}\right)[[t]] .
$$

It is well known that for every prime number $l \neq p$ there is a lisse, rankone, $l$-adic étale sheaf $\mathcal{L}_{\Psi}(f)$ on $V$ whose associated $L$-function is identical to $L(V, f ; t)$. By Grothendieck's Lefschetz trace formula and Deligne's fundamental theorem, if

$$
H_{c}^{i}\left(V \otimes_{k} \bar{k}, \mathcal{L}_{\Psi}(f)\right)=0 \text { for } i \neq n,
$$

then one obtains the estimate

$$
\left|S_{r}(V, f)\right| \leq\left(\operatorname{dim} H_{c}^{n}\left(V \otimes_{k} \bar{k}, \mathcal{L}_{\Psi}(f)\right)\right) \sqrt{q}^{r n}
$$

(where $H_{c}^{i}\left(V \otimes_{k} \bar{k}, \mathcal{L}_{\Psi}(f)\right)$ denotes $l$-adic cohomology with proper supports). When $V=\left(\mathbf{G}_{m}\right)^{n}$ or $\mathbf{A}^{n}$, we shall give conditions on $f$ that allow us to deduce (5) and give a simple formula for $\operatorname{dim} H_{c}^{n}\left(V \otimes_{k} \bar{k}, \mathcal{L}_{\Psi}(f)\right)$.

Consider first the case $V=\left(\mathbf{G}_{m}\right)^{n}$. The regular functions on $V$ defined over $k$ are the Laurent polynomials with coefficients in $k$, i.e., elements of $k\left[x_{1}, x_{1}^{-1}, \ldots, x_{n}, x_{n}^{-1}\right]$. For $j=\left(j_{1}, \ldots, j_{n}\right) \in \mathbf{Z}^{n}$, let $x^{j}=x_{1}^{j_{1}} \cdots x_{n}^{j_{n}}$. A Laurent polynomial $f$ over $k$ can be written

$$
f=\sum_{j \in J} a_{j} x^{j}
$$

where $J$ is a finite subset of $\mathbf{Z}^{n}$ and $a_{j} \in k^{\times}$. We define the Newton polyhedron $\Delta(f)$ of $f$ to be the convex closure in $\mathbf{R}^{n}$ of the set $J \cup\{(0, \ldots, 0)\}$. For each face $\sigma$ of $\Delta(f)$, define a Laurent polynomial $f_{\sigma}$ by

$$
f_{\sigma}=\sum_{j \in \sigma \cap J} a_{j} x^{j}
$$

Call $f$ nondegenerate with respect to $\Delta(f)$ (Kouchnirenko [6]) if for every face $\sigma$ of $\Delta(f)$ that does not contain the origin, $\partial f_{\sigma} / \partial x_{1}, \ldots, \partial f_{\sigma} / \partial x_{n}$ have no common zero in $\left(\bar{k}^{\times}\right)^{n}$. The set of all nondegenerate polynomials having a given Newton polyhedron is Zariski open in the set of all polynomials having that Newton polyhedron, except possibly if the characteristic of $k$ lies in a certain finite set which depends on the Newton polyhedron. We define the dimension of $\Delta(f)$ to be the dimension of the smallest subspace of $\mathbf{R}^{n}$ containing $\Delta(f)$. Let $V(f)$ denote the volume of $\Delta(f)$ with respect to Lebesgue measure on $\mathbf{R}^{n}$.

THEOREM 1. Let $\Delta$ be an $n$-dimensional convex polyhedron in $\mathbf{R}^{n}$ with vertices in $\mathbf{Z}^{n}$ that contains the origin. There is a finite set of rational primes $S_{\Delta}$ such that the following holds: If $\operatorname{char}(k) \notin S_{\Delta}, f \in k\left[x_{1}, x_{1}^{-1}, \ldots, x_{n}, x_{n}^{-1}\right]$ with $\Delta(f)=\Delta$, and $f$ is nondegenerate with respect to $\Delta(f)$, then

(i) $H_{c}^{i}\left(\left(\mathbf{G}_{m}\right)^{n} \otimes_{k} \bar{k}, \mathcal{L}_{\Psi}(f)\right)=0$ if $i \neq n$;

(ii) $\operatorname{dim} H_{c}^{n}\left(\left(\mathbf{G}_{m}\right)^{n} \otimes_{k} \bar{k}, \mathcal{L}_{\Psi}(f)\right)=n ! V(f)$. 
If in addition the origin is an interior point of $\Delta$, then

(iii) $H_{c}^{n}\left(\left(\mathbf{G}_{m}\right)^{n} \otimes_{k} \bar{k}, \mathcal{L}_{\Psi}(f)\right)$ is pure of weight $n$.

COROLLARY. Under the hypotheses of Theorem 1,

$$
\left|S\left(\left(\mathbf{G}_{m}\right)^{n}, f\right)\right| \leq n ! V(f) \sqrt{q}^{n} .
$$

ProOF. Using the ideal theory of [6], we are able to develop a cohomology theory along the lines of $[\mathbf{4}]$ and $[\mathbf{5}]$ to show that $L\left(\left(\mathbf{G}_{m}\right)^{n}, f ; t\right)^{(-1)^{n-1}}$ is a polynomial of degree $n ! V(f)$ and obtain $p$-adic estimates for its roots. The proof then proceeds by induction on $n$. After an invertible change of coordinates, one may regard $f$ as a one-parameter family of Laurent polynomials in $n-1$ variables, each satisfying the induction hypothesis and containing the origin in the interior of its Newton polyhedron. Applying basic theorems of $l$-adic cohomology shows that $H_{c}^{i}=0$ except possibly in dimensions $n$ and $n+1$. Corollaire 1.4.4 of $[3]$ and the fact that $L\left(\left(\mathbf{G}_{m}\right)^{n}, f ; t\right)^{(-1)^{n-1}}$ is a polynomial show that $H_{c}^{n+1}=0$. The $p$-adic estimate for the roots, Deligne's fundamental theorem [3], and the product formula for valuations then imply purity.

We conjecture that Theorem 1 remains true without restriction on the characteristic of $k$. This can be verified if $n=2$ and in many other cases (see the examples at the end of this note).

We now turn to the case $V=\mathbf{A}^{n}, f \in k\left[x_{1}, \ldots, x_{n}\right]$. Since an ordinary polynomial may also be regarded as a Laurent polynomial, all our previous definitions concerning the Newton polyhedron make sense in this context. We call $f \in k\left[x_{1}, \ldots, x_{n}\right]$ commode if for each $i=1, \ldots, n, f$ contains a term $\gamma_{i} x_{i}^{d_{i}}$ with $\gamma_{i} \in k^{\times}, d_{i}>0$. For each subset $A \subseteq\{1, \ldots, n\}$, let $X_{A}$ be the subspace of $\mathbf{R}^{n}$ where $x_{i}=0$ for all $i \notin A$. Let $V_{A}(f)$ be the volume of $\Delta(f) \cap X_{A}$, computed with respect to Lebesgue measure on $X_{A}$ normalized so that a fundamental domain for $\mathbf{Z}^{n} \cap X_{A}$ has volume 1 . Let $|A|$ denote the cardinality of $A$. Define the Newton number $\nu(f)$ by the formula

$$
\nu(f)=\sum_{A \subseteq\{1, \ldots, n\}}(-1)^{n-|A|}|A| ! V_{A}(f) .
$$

Let $\mathbf{R}_{+}$denote the nonnegative real numbers.

THEOREM 2. Let $\Delta$ be a convex polyhedron in $\left(\mathbf{R}_{+}\right)^{n}$ with vertices in $\mathbf{Z}^{n}$ that has a vertex at the origin and on each of the coordinate axes. There is a finite set of rational primes $S_{\Delta}$ such that the following holds: If $\operatorname{char}(k) \notin S_{\Delta}$, $f \in k\left[x_{1}, \ldots, x_{n}\right]$ with $\Delta(f)=\Delta$, and $f$ is nondegenerate with respect to $\Delta(f)$, then $L\left(\mathbf{A}^{n}, f ; t\right)^{(-1)^{n-1}}$ is a polynomial of degree $\nu(f)$, all of whose reciprocal roots are algebraic integers pure of weight $n$.

COROLlaRY. Under the hypotheses of Theorem $2,\left|S\left(\mathbf{A}^{n}, f\right)\right| \leq \nu(f) \sqrt{q}^{n}$.

ProOF. The fact that $L\left(\mathbf{A}^{n}, f ; t\right)^{(-1)^{n-1}}$ is a polynomial is a consequence of the $p$-adic theory. Theorem 2 then follows from Theorem 1 by the standard relations between exponential sums over $\mathbf{A}^{n}$ and $\left(\mathbf{G}_{m}\right)^{n}$.

We conjecture that Theorem 2 remains true without restriction on the characteristic of $k$. This can be verified if $n=2$ and in many other cases 
(see Theorem 3 below). Of course, we believe that there is a cohomological explanation for this result:

CONJECTURE. If $f \in k\left[x_{1}, \ldots, x_{n}\right]$ is commode and nondegenerate with respect to $\Delta(f)$, then

(i) $H_{c}^{i}\left(\mathbf{A}^{n} \otimes_{k} \bar{k}, \mathcal{L}_{\Psi}(f)\right)=0$ if $i \neq n$;

(ii) $\operatorname{dim} H_{c}^{n}\left(\mathbf{A}^{n} \otimes_{k} \bar{k}, \mathcal{L}_{\Psi}(f)\right)=\nu(f)$;

(iii) $H_{c}^{n}\left(\mathbf{A}^{n} \otimes_{k} \bar{k}, \mathcal{L}_{\Psi}(f)\right)$ is pure of weight $n$.

We can prove this conjecture provided $\Delta(f)$ has a somewhat special form.

THEOREM 3. Suppose $f \in k\left[x_{1}, \ldots, x_{n}\right]$ is commode and nondegenerate with respect to $\Delta(f)$. Assume in addition that for each codimension-one face $\sigma$ of $\Delta(f)$ that does not contain the origin, all coordinates of the exterior normal vector to $\sigma$ with respect to the standard basis are positive (where the exterior normal vector is the one pointing out of $\Delta(f))$. Then all conclusions of the Conjecture hold. In particular, we have

$$
\left|S\left(\mathbf{A}^{n}, f\right)\right| \leq \nu(f) \sqrt{q}^{n} .
$$

PROOF. The proof is identical to the proof of Theorem 1, the point being that one can simply specialize one of the variables to regard $f$ as a oneparameter family of polynomials, each satisfying the induction hypothesis.

EXAMPLES. The Laurent polynomial

$$
f=\gamma_{1} x_{1}^{d_{1}}+\cdots+\gamma_{n} x_{n}^{d_{n}}+\frac{\gamma_{n+1}}{x_{1}^{e_{1}} \ldots x_{n}^{e_{n}}}
$$

where the $\gamma_{i}$ lie in $k^{\times}$and the $d_{i}$ and $e_{j}$ are positive integers prime to $p$, satisfies the hypotheses of Theorem 1 (one can show in addition that no restriction on $\operatorname{char}(k)$ is necessary) and $n ! V(f)=\left(\prod_{i=1}^{n} d_{i}\right)\left(1+\sum_{i=1}^{n} e_{i} / d_{i}\right)$. Thus

$$
\begin{aligned}
\mid S\left(\left(\mathbf{G}_{m}\right)^{n}, \gamma_{1} x_{1}^{d_{1}}\right. & \left.+\cdots+\gamma_{n} x_{n}^{d_{n}}+\frac{\gamma_{n+1}}{x_{1}^{e_{1}} \cdots x_{n}^{e_{n}}}\right) \mid \\
& \leq\left(\prod_{i=1}^{n} d_{i}\right)\left(1+\sum_{i=1}^{n} \frac{e_{i}}{d_{i}}\right) \sqrt{q}^{n} .
\end{aligned}
$$

See Carpentier [1] for a $p$-adic study of this exponential sum.

Consider the polynomial

$$
f\left(x_{1}, \ldots, x_{n}\right)=\gamma_{1} x_{1}^{d_{1}}+\cdots+\gamma_{n} x_{n}^{d_{n}}+g\left(x_{1}, \ldots, x_{n}\right),
$$

where $g$ is chosen subject to the restrictions that $\Delta(f)$ be the simplex with vertices at the origin and at $\left(d_{1}, 0, \ldots, 0\right), \ldots,\left(0, \ldots, 0, d_{n}\right)$ and that $f$ be nondegenerate with respect to $\Delta(f)$. Then $f$ satisfies the hypotheses of Theorem 3 and $\nu(f)=\prod_{i=1}^{n}\left(d_{i}-1\right)$, hence

$$
\left|S\left(\mathbf{A}^{n}, f\right)\right| \leq\left(\prod_{i=1}^{n}\left(d_{i}-1\right)\right) \sqrt{q}^{n} .
$$

It can be shown that this result includes Deligne's theorem [2, Théorème 8.4] as the special case where $d_{1}=\cdots=d_{n}$. 


\section{REFERENCES}

1. M. Carpentier, $p$-Adic cohomology of generalized hyperkloosterman sums, Doctoral thesis, University of Minnesota (August, 1985).

2. P. Deligne, La conjecture de Weil. I, Publ Math. I.H.E.S. 43 (1974), 273-307.

3. _, La conjecture de Weil. II, Publ. Math. I.H.E.S. 52 (1980), 137-252.

4. B. Dwork, On the zeta function of a hypersurface, Publ. Math. I.H.E.S. 12 (1962), $5-68$.

5. _- On the zeta function of a hypersurface. II, Ann. of Math. 80 (1964), 227-299.

6. A. G. Kouchnirenko, Polyèdres de Newton et nombres de Milnor, Invent. Math. 32 (1976), 1-31.

7. S. Sperber and A. Adolphson, Newton polyhedra and the degree of the L-function associated to an exponential sum, Invent. Math. (to appear).

8. - p-Adic estimates for exponential sums and the theorem of Chevalley-Warning, Ann. Sci. École Norm. Sup. (to appear).

DePARTMENT OF MATHEMATICS, OKLAHOMA STATE UNIVERSiTy, STILlWATER, OKLAHOMA 74078

SCHOOL OF MATHEMATICS, UNIVERSity OF MinNesota, MinNEAPOlis, MiNNESOTA 55455 